\title{
Crecimiento, rendimiento y rentabilidad del maíz VS-535 en función del biofertilizante y nitrógeno
}

\section{Growth, performance and profitability of VS-535 corn based on biofertilizer and nitrogen}

\author{
Cid Aguilar-Carpio ${ }^{1 *}$, José Alberto Salvador Escalante-Estrada ${ }^{1}$, Immer Aguilar-Mariscal $^{2}$, Adriana \\ Perez-Ramirez ${ }^{3}$ \\ ${ }^{1}$ Programa de Botánica. Campus Montecillo, Colegio de Postgraduados. Km 36.5 Carretera México-Texcoco. CP. 56230. \\ Montecillo, Edo. de México, México. \\ ${ }^{2}$ Facultad de Biología. Universidad Autónoma del Estado de Morelos. Cuernavaca, Morelos, México. \\ ${ }^{3}$ IDAGRO S. de R. L. de C. V. Carretera Yautepec-Tlayacapan S/N. Col. Puente Pantitlan. CP. 62545. Tlayacapan, Morelos, \\ México. \\ ${ }^{*}$ Autor de correspondencia: aguilar.cid@colpos.mx
}

Artículo científico recibido: 21 de enero de 2016 aceptado: 14 de marzo de 2017

RESUMEN. El objetivo del estudio fue determinar el efecto del biofertilizante y del nitrógeno en la variedad sintética de maíz VS-535 sobre la ocurrencia de las fases fenológicas, análisis de crecimiento, rendimiento, componentes, eficiencia en el uso del agua, contenido de nitrógeno y la rentabilidad. La siembra se realizó el 10 de julio de 2012, los tratamientos fueron la inoculación de biofertilizante y la aplicación de 0,80 y $160 \mathrm{~kg} \mathrm{ha}^{-1}$ de nitrógeno. Se contabilizó el número de hojas y el área foliar, con lo que se calculó el índice (IAF) y la duración de área foliar (DAFT). También se determinó el contenido de materia seca (MS), las tasa de crecimiento del cultivo $(\overline{T C C})$, y la tasa de asimilación neta $(\overline{T A N})$. En madurez fisiológica se evaluó el contenido de materia seca (MS) y el rendimiento de grano (RG), se calculó la eficiencia en el uso del agua y del nitrógeno, y se realizó un análisis económico. Con $160 \mathrm{~kg} \mathrm{ha}^{-1}$ de nitrógeno se tuvo el mayor IAF y DAFT. Para MS, EUA, EAN, $\overline{T C C}$, mientras que las mayores $\overline{T A N}$ se tuvieron con biofertilizante y nitrógeno a 80 y $160 \mathrm{~kg} \mathrm{ha}^{-1}$. Para RG la mayor respuesta se encontró con biofertilizante y nitrógeno a $160 \mathrm{~kg} \mathrm{ha}^{-1}$, lo que genero la mayor rentabilidad del cultivo.

Palabras clave: Área foliar, fenología, grano, nutrición, variedad sintética

ABSTRACT. The objective of the study was to determine the effect of biofertilizer and nitrogen on the synthetic variety of VS-535 corn, on the occurrence of phenological phases, growth analysis, performance, components, water use efficiency, nitrogen content and profitability. The sowing was performed on July 10, 2012, the treatments were the inoculation of biofertilizer and the application of 0,80 , and $160 \mathrm{~kg} \mathrm{ha}^{-1}$ of nitrogen. The number of leaves and leaf area were counted, calculating the index (LAI) and leaf area duration (TLAD). The dry matter (DM) content was also determined, as well as the crop growth rate $(\overline{C G R})$ and the net assimilation rate $(\overline{N A R})$ was also evaluated. On physiological maturity, the dry matter (DM) content (DM) and grain performance (GP) were evaluated; the efficiency in the use of water and nitrogen was calculated, and an economic analysis was performed. It had the highest LAI and TLAD with $160 \mathrm{~kg} \mathrm{ha}^{-1}$ of nitrogen. For DM, EUA, EAN, $\overline{C G R}$, while the older $\overline{N A R}$ were with biofertilizer and nitrogen at 80 and $160 \mathrm{~kg} \mathrm{ha}^{-1}$. For GP, the highest response was found with biofertilizer and nitrogen at $160 \mathrm{~kg}$ ha-1, which generated the highest profitability of the crop.

Key words: Leaf area, phenology, grain, nutrition, synthetic variety 


\section{INTRODUCCIÓN}

El maíz es uno de los cultivos más importantes a nivel mundial, para los mexicanos constituye la base de la alimentación, aportando energía y proteínas (Domínguez et al. 2014). Los principales estados productores de maíz en México son Sinaloa, Jalisco, Estado de México, Michoacán, Guanajuato y Chihuahua; mientras que el estado de Guerrero, ocupa el séptimo lugar en producción, con rendimiento promedio de $2.79 \mathrm{t} \mathrm{ha}^{-1}$ (SIAP 2016). A pesar de ser uno de los principales estados productores, sus rendimientos promedios son bajos, debido al uso de genotipos criollos, aunado a sus tierras con bajo potencial productivo. Por lo que, el uso de semilla mejorada, como las variedades sintéticas es necesario para lograr su máximo potencial productivo (Espinosa et al. 2003).

En la agricultura se requiere hacer uso eficiente del agua (EUA) y nitrógeno $(\mathrm{N})$, debido a que son dos de los insumos más importantes para la producción, los cuales deben estar bien provistos en cantidad y oportunidad para asegurar un estado fisiológico óptimo en la floración, momento en que se determina el rendimiento (Andrade et al. 2002). Por otro lado, la disponibilidad de nitrógeno en el suelo influye en las tasas de crecimiento del maíz, herramienta que permite analizar el comportamiento de distintos cultivares, niveles de fertilización y condiciones ambientales (D'Andrea et al. 2008). La incorporación de $\mathrm{N}$ en el suelo es importante, debido al bajo contenido de los suelos agrícolas; por lo que su aplicación en forma fraccionada, estimula el incremento del rendimiento (Villafaña et al. 2014). Investigaciones realizadas en el maíz con la aplicación de nitrógeno, han incrementado el crecimiento, rendimiento de grano y la eficiencia agronómica del nitrógeno y agua (Yu-Kui et al. 2009, Mamani-Pati et al. 2010).

Por otra parte, la inoculación de hongos y bacterias representan una alternativa que promueve el crecimiento, nutrición y sanidad de la planta (Smith y Read 2008). Múltiples trabajos han mostrado que los biofertilizantes influyen en el desarrollo y crecimiento de los cultivos, y el rendimiento de grano en maíz (Uribe y Dzib 2006, Díaz et al. 2008). Al respecto Díaz et al. (2008), reportaron que al inocular la semilla de maíz con Glomus intraradices (biofertilizante), se tienen rendimientos similares al testigo con fertilización inorgánica; esta misma cepa ha promovido de forma significativa la producción de forraje fresco y de grano (Pecina et al. 2005, Uribe y Dzib 2006). La respuesta en el crecimiento y rendimiento del grano de maíz a la fertilización nitrogenada puede variar en función del nivel inicial de nitrógeno en el suelo, el biofertilizante y el ambiente de desarrollo (López et al. 2007). Por lo que, el objetivo del estudio fue determinar el efecto del biofertilizante y nitrógeno en el maíz VS-535 sobre la ocurrencia de las fases fenológicas, análisis de crecimiento, rendimiento y sus componentes, eficiencia en el uso del agua, contenido de nitrógeno y la rentabilidad en maíz, en siembra de temporal.

\section{MATERIALES Y MÉTODOS}

El estudio se estableció el 10 de julio de 2012 bajo condiciones de temporal en Iguala, Guerrero, que tiene clima cálido subhúmedo con lluvias en verano, precipitación anual de $1100 \mathrm{~mm}$ y altitud de 635 m (García 2005).

Se utilizó la variedad sintética de maíz VS535, los tratamientos consistieron en la inoculación a la semilla con biofertilizante formado con $1 \mathrm{~kg}$ de Glomus intraradices y $0.4 \mathrm{~kg}$ de Azospirillum brasilense, que se mezcló con agua y adherente en $20 \mathrm{~kg}$ de semilla de maíz. Se aplicaron tres niveles de fertilización 0,80 y $160 \mathrm{~kg} \mathrm{ha}^{-1}$ de nitrógeno $(\mathrm{N})$, el cual se aplicó de forma fraccionada, la mitad al momento de la siembra y el resto a los $30 \mathrm{~d}$ después de la siembra (dds). La densidad de población fue de $6.25 \mathrm{pl} \mathrm{m}^{-2}$, con distancia entre surcos de $80 \mathrm{~cm}$. Se utilizó un diseño experimental de bloques completos al azar con cuatro repeticiones. La unidad experimental estuvo formada por cuatro surcos de $0.8 \mathrm{~m}$ de ancho por $4 \mathrm{~m}$ de largo. La temperatura máxima (Tmax) y mínima (Tmin) decenal, y la precipitación (PP, $\mathrm{mm}$ ) durante el desarrollo del estudio, se obtuvo del Servicio Meteorológico Nacional 
(CONAGUA). También se registró la ocurrencia de las fases fenológicas como: días a emergencia (E), días a floración (FL) y días a madurez fisiológica (MF) de acuerdo al criterio de Ritchie y Hanway (1982). Para cada fase fenológica se determinó la acumulación de unidades calor (UC, ${ }^{\circ} \mathrm{C} d$ ) para el cultivo, mediante el método residual (Snyder 1985), con la siguiente ecuación:

$$
U C=\frac{T \max +T \min }{2}-T B
$$

Dónde: $U C=$ unidades Calor $\left({ }^{\circ} \mathrm{C} d\right)$, Tmax $=$ temperatura máxima diaria $\left({ }^{\circ} \mathrm{C}\right)$, Tmin = temperatura mínima diaria $\left({ }^{\circ} \mathrm{C}\right)$ y $\mathrm{TB}=$ la temperatura base, considerada como $10^{\circ} \mathrm{C}$ (Iñiguez-Covarrubias et al. 2014).

La evapotranspiración del cultivo (ETc) se calculó con los datos de la evaporación (Ev) del tanque tipo " $\mathrm{A}$ ", utilizando 0.6 como coeficiente para el evaporímetro, $\mathrm{kc}$ inicial $=0.35$, kc a mediados del periodo $=1.14$ y $\mathrm{kc}$ final $=0.6$ (Doorenbos y Pruitt 1986), con la siguiente ecuación:

$$
E T c=E v \times 0.6 \times K c
$$

Para evaluar el crecimiento del cultivo, se hicieron muestreos destructivos de dos plantas en la parcela útil de cada unidad experimental a los 31, 65 y 105 dds, en cada uno de los muestreos se contabilizó el número de hojas por $\mathrm{m}^{2}(\mathrm{NH})$ y el área foliar se midió con un integrador electrónico (LI-COR 3100). Se calculó el índice de área foliar (IAF) y duración del área foliar total (DAFT), con las siguientes ecuaciones:

$$
I A F=\frac{\left(\frac{A F}{N P}\right) * D P}{100 d m^{2}}
$$

Dónde: $\mathrm{AF}=$ área foliar $\left(\mathrm{dm}^{2}\right), \mathrm{NP}=$ número de plantas muestreadas y $\mathrm{DP}=$ número de plantas $\mathrm{m}^{-2}$.

$$
D A F=\sum \frac{\left(I A F_{1}+I A F_{2}\right) *\left(T_{2}-T_{1}\right)}{2}
$$

Dónde: $\Sigma=$ sumatoria de la duración del área foliar para cada periodo estudiado, $\mathrm{IAF}_{1}=$ índice de área foliar en un tiempo inicial ( $T_{1}$, días), $I A F_{2}=$ índice de área foliar en un tiempo final ( $T_{2}$, días) (Aguilar et al. 2015a). También se evaluó la materia seca (MS, $\mathrm{g} \mathrm{m}^{-2}$ ); para lo cual las muestras se secaron a $80^{\circ} \mathrm{C}$ en una estufa de circulación de aire forzado hasta peso constante. Con estos datos se calcularon las tasas medias de: crecimiento del cultivo $(\overline{T C C})$ y asimilación neta $(\overline{T A N})$, mediante las ecuaciones:

$$
(\overline{T C C})=\frac{\left(P S_{2}-P S_{1}\right)}{A\left(T_{2}-T_{1}\right)}
$$

Dónde: $\mathrm{PS}_{2}$ y $\mathrm{PS}_{1}$ indican el peso de la materia seca de la planta en los tiempos $T_{2}$ y $T_{1}$, respectivamente y $\mathrm{A}$ representa el área ocupada por la planta.

$$
\overline{T A N}=\frac{\left(P S_{2}-P S_{1}\right)}{\left(I A F_{2}-I A F_{1}\right)} / \frac{\left(L n I A F_{2}-L n I A F_{1}\right)}{\left(T_{2}-T_{1}\right)}
$$

Dónde: $\mathrm{PS}_{2}$ y $\mathrm{PS}_{1}$ expresan el peso de $\mathrm{MS}$ de la planta, $L n \mathrm{IAF}_{2}$ y $\mathrm{Ln} \mathrm{IAF}_{1}$, logaritmo natural del índice de área foliar en el tiempo $\mathrm{T}_{2}$ y $\mathrm{T}_{1}$, respectivamente (Aguilar et al. 2015a). A madurez fisiológica (MF) se determinó la producción de biomasa total en base a materia seca (MS total, $\mathrm{g} \mathrm{m}^{-2}$ ), índice de cosecha (IC, \%), rendimiento de grano ( $R G, 10 \%$ de humedad, $\mathrm{g} \mathrm{m}^{-2}$ ), peso de 100 granos (P100G, g), número de granos (NG, $\mathrm{m}^{2}$ ), número de hileras $(\mathrm{NH})$ y número de granos por hilera (NGH). Se calculó la eficiencia en el uso del agua del RG (EUARG, $\mathrm{g} \mathrm{m}^{-2} \mathrm{~mm}^{-1}$ ) y $\mathrm{MS}$ (EUAMS, $\mathrm{g} \mathrm{m}^{-2} \mathrm{~mm}^{-1}$ ) con la ecuación:

$$
E U A=R G o M S / E T c
$$

Dónde: $E U A$ = Eficiencia en el uso del agua, RG $=$ Rendimiento de grano $\left(\mathrm{g} \mathrm{m}^{-2}\right), \mathrm{MS}=$ Materia seca $\left(\mathrm{g} \mathrm{m}^{-2}\right)$, ETc $=$ Evapotranspiración del cultivo $(\mathrm{mm})$ ocurrida durante el ciclo del cultivo (Escalante 1995) y la eficiencia agronómica del N (EAN, $\mathrm{g} \mathrm{g}^{-1}$ de $\mathrm{N}$ aplicado), considerado como el incremento en el rendimiento por unidad de fertilizante aplicado, se calculó a través de la siguiente ecuación:

$$
E A N=\frac{(R G N-R G S N)}{N A}
$$

Dónde: $\mathrm{RGN}=$ rendimiento de grano con nitrógeno 


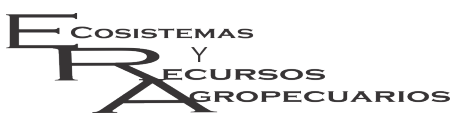

$\left(\mathrm{g} \mathrm{m}^{-2}\right)$, RGSN $=$ rendimiento de grano $\sin \mathrm{ni}-$ trógeno $\left(\mathrm{g} \mathrm{m}^{-2}\right)$ y $\mathrm{NA}=$ cantidad de nitrógeno aplicado $\left(\mathrm{g} \mathrm{m}^{-2}\right)$ (Fageria y Baligar 2005).

A las variables en estudio, se les aplicó un análisis de varianza (ANDEVA), con el programa estadístico de SAS (Statistical Analysis System, Versión 9.0) y la prueba de comparación de medias de Tukey $(\alpha=0.05)$. Adicionalmente, se realizó un análisis económico para rendimiento, utilizando las siguientes ecuaciones:

$$
I N=Y P y-\sum(X i P i+C P)
$$

Donde IN $=$ Ingreso neto, $\mathrm{Y}=$ Rendimiento $(\mathrm{kg}$ $\mathrm{ha}^{-1}$ ), Py $=$ precio por $\mathrm{kg}, \quad \Sigma \mathrm{XiPi}=$ suma de costos variables, CF $=$ costos fijos (Volke 1982). También se determinó la GPI con la ecuación:

$$
G P I=\frac{\left(\sum X i P i+C F\right)}{Y P y}
$$

Dónde: $\mathrm{GPI}=$ ganancia por peso invertido, $\mathrm{Y}=$ Rendimiento $\left(\mathrm{kg} \mathrm{ha}^{-1}\right), \mathrm{Py}=$ precio por $\mathrm{kg}, \Sigma \mathrm{XiPi}$ = suma de costos variables, $\mathrm{CF}=$ costos fijos (Volke 1982)

\section{RESULTADOS}

Fenología, condiciones climáticas, unidades calor y evapotranspiración

En la Tabla 1 , se observa la media decenal de la Tmin y Tmax durante el desarrollo del cultivo que fluctuó entre 24 y $34{ }^{\circ} \mathrm{C}$, respectivamente. De la siembra (S) a la $\mathrm{FL}$, la Tmin y Tmax promedio fue de 24 y $33{ }^{\circ} \mathrm{C}$, mientras que de la $\mathrm{FL}$ a la MF fue de 24 a $34{ }^{\circ} \mathrm{C}$, respectivamente. La suma de la PP durante el ciclo del cultivo fue de $558 \mathrm{~mm}$. La mayor PP (417 mm) ocurrió en la etapa de S a $\mathrm{FL}$, en los meses de julio, agosto y septiembre. Sin embargo, de FL a MF la PP fue de $157 \mathrm{~mm}$. La emergencia ocurrió a los 5 dds con un requerimiento de calor de $60^{\circ} \mathrm{C}$ d, la floración ocurrió a los $65 \mathrm{dds}$ con $1952{ }^{\circ} \mathrm{C} \mathrm{d}$ y la MF a los $105 \mathrm{dds}\left(3096^{\circ} \mathrm{C} \mathrm{d}\right)$ (Tabla 1). Para la ETc, de la S a la E fue de 18 $\mathrm{mm}$, de la E a la FL de 231 y de FL a la MF de 178 $\mathrm{mm}$, con ETc total de $409 \mathrm{~mm}$.
Aguilar-Carpio et al. Análisis de crecimiento en maíz Ecosist. Recur. Agropec. 4(12):475-483,2017

Número de hojas, índice de área foliar y duración del área foliar

EI ANDEVA mostró diferencias significativas para el IAF y la DAFT, debido a la interacción Biofertilizante $\times \mathrm{N}$ (Tabla 2). En general, se observó que el cultivo sin biofertilizante y $160 \mathrm{~kg} \mathrm{ha}^{-1}$ de nitrógeno presentó la más alta respuesta en el IAF y DAFT a los 75 dds. En contraste, el maíz con Biofertilizante presentó mayor IAF y DAFT, con $160 \mathrm{~kg} \mathrm{ha}^{-1}$ de nitrógeno.

Tasa de crecimiento del cultivo $(\overline{T C C})$, asimilación neta $(\overline{T A N})$ y materia seca total (MS)

En el análisis de la $\overline{T C C}, \overline{T A N}$ y MS para la interacción Biofertilizante $\times \mathrm{N}$, se observaron diferencias significativas (Tabla 3). La $\overline{T C C}$ más alta se presentó con la aplicación de Biofertilizante y nitrógeno a 80 y $160 \mathrm{~kg} \mathrm{ha}^{-1}$ desde los 20 hasta los 105 dds. La mayor $\overline{T A N}$ ocurrió a los 20 dds y decreció durante el ciclo de cultivo hasta los 105 dds. La aplicación de Biofertilizante con 80 y $160 \mathrm{~kg} \mathrm{ha}^{-1}$ de nitrógeno, tuvieron las mayores $\overline{T A N}$ durante el ciclo de cultivo. Con respecto a la mayor producción de MS, esta se presentó con Biofertilizante y nitrógeno a 80 y $160 \mathrm{~kg} \mathrm{ha}^{-1}$.

\section{Índice de cosecha (IC), rendimiento (RG) y sus} componentes

En cuanto al RG se observó que la respuesta al nitrógeno fue en función del biofertilizante. EI mayor RG se observó con la aplicación de $160 \mathrm{~kg}$ $\mathrm{ha}^{-1}$ de nitrógeno más el biofertilizante, lo cual se relaciona con el mayor número de granos (Tabla 4).

\section{Eficiencia en el uso del agua (EUA)}

La EUAMS y EUARG mostró diferencias significativas debido a la interacción Biofertilizante $x$ N. La EUAMS más alta se encontró en Biofertilizante con 80 y $160 \mathrm{~kg} \mathrm{ha}^{-1}$ de nitrógeno (Tabla 5). La mayor EUARG, se observó con Biofertilizante y con $160 \mathrm{~kg} \mathrm{ha}^{-1}$ de $\mathrm{N}$.

\section{Eficiencia agronómica del nitrógeno (EAN)}

En la Tabla 5 se observa que la EAN más alta correspondió a la aplicación de biofertilizante 
Tabla 1. Medias decenales de la temperatura máxima (Tmax), mínima (Tmin), suma decenal de la precipitación (PP), evapotranspiración (ETc) y unidades calor (UC), durante el ciclo del cultivo del maíz VS-535.

\begin{tabular}{cccccccc}
\hline Meses & $10 \mathrm{~d}$ & $\mathrm{Tmax}\left({ }^{\circ} \mathrm{C}\right)$ & $\mathrm{Tmin}\left({ }^{\circ} \mathrm{C}\right)$ & $\mathrm{PP}(\mathrm{mm})$ & $\mathrm{UC}\left({ }^{\circ} \mathrm{C}\right)$ & $\mathrm{ETc}(\mathrm{mm})$ & Fenología \\
\hline Julio & 1 & 34 & 26 & 29 & 262 & 19 & \\
Julio & 2 & 33 & 25 & 60 & 512 & 37 & $\mathrm{~S}-\mathrm{E}$ \\
Julio & 3 & 33 & 25 & 84 & 794 & 55 & \\
Agosto & 4 & 33 & 25 & 46 & 1050 & 72 & \\
Agosto & 5 & 32 & 24 & 43 & 1298 & 121 & \\
Agosto & 6 & 32 & 24 & 168 & 1570 & 177 & \\
Septiembre & 7 & 33 & 25 & 16 & 1827 & 224 & \\
Septiembre & 8 & 33 & 25 & 37 & 2078 & 268 & $\mathrm{FL}$ \\
Septiembre & 9 & 33 & 25 & 24 & 2332 & 305 & \\
Octubre & 10 & 32 & 24 & 55 & 2575 & 345 & \\
Octubre & 11 & 34 & 26 & 1 & 2836 & 388 & $\mathrm{MF}$ \\
Octubre & 12 & 34 & 26 & 24 & 3096 & 409 & \\
\hline S = Siembra, E = Emergencia, $\mathrm{FL}=$ Floración, MF = Madurez fisiológica.
\end{tabular}

Tabla 2. Número de hojas (NH), índice de área foliar (IAF) y duración del área foliar total (DAFT) del maíz en función del biofertilizante y nitrógeno $(\mathrm{N})$.

\begin{tabular}{|c|c|c|c|c|c|c|c|c|}
\hline \multirow{2}{*}{ Biofertilizante } & \multirow{2}{*}{$\mathrm{N}\left(\mathrm{kg} \mathrm{ha}^{-1}\right)$} & \multicolumn{3}{|c|}{$\mathrm{NH}\left(\mathrm{m}^{2}\right)$} & \multicolumn{3}{|c|}{$\mathrm{IAF}$} & \multirow{2}{*}{ DAFT } \\
\hline & & 31 & 75 & 105 & 31 & 75 & 105 & \\
\hline & \multicolumn{8}{|c|}{ Días después de la siembra } \\
\hline & 0 & $53^{a}$ & $87^{a}$ & $81^{a}$ & $1.4^{d}$ & $4.2^{b}$ & $2.4^{b c}$ & $253^{b}$ \\
\hline \multirow[t]{3}{*}{$\operatorname{Sin}$} & 80 & $56^{a}$ & $94^{a}$ & $90^{a}$ & $1.6^{c}$ & $4.4^{a}$ & $2.8^{b}$ & $258^{b}$ \\
\hline & 160 & $59^{a}$ & $95^{a}$ & $91^{a}$ & $1.8^{b}$ & $4.8^{a}$ & $3.7^{a}$ & $299^{a}$ \\
\hline & 0 & $54^{a}$ & $91^{a}$ & $78^{a}$ & $1.4^{d}$ & $3.3^{c}$ & $1.9^{c}$ & $203^{c}$ \\
\hline \multirow[t]{2}{*}{ Con } & 80 & $67^{a}$ & $93^{a}$ & $87^{a}$ & $1.8^{b}$ & $4.0^{b}$ & $2.8^{b}$ & $255^{b}$ \\
\hline & 160 & $67^{a}$ & $97^{a}$ & $87^{a}$ & $2.1^{a}$ & $4.4^{a}$ & $3.1^{b}$ & $286^{a b}$ \\
\hline \multicolumn{2}{|c|}{ Tukey $\alpha=0.05$ (DMS) } & 15 & 10 & 10 & 0.2 & 0.4 & 0.9 & 33 \\
\hline \multicolumn{2}{|c|}{ Media general } & 72 & 72 & 43 & 1.7 & 4.2 & 2.8 & 259 \\
\hline \multicolumn{2}{|c|}{ CV. (\%) } & 3 & 3 & 6 & 3 & 7 & 9 & 6 \\
\hline
\end{tabular}

Tabla 3. Tasa de crecimiento del cultivo $(\overline{T C C})$, asimilación neta $(\overline{T A N})$ y materia seca total (MS) en el maíz VS-535 en función del biofertilizante y nitrógeno $(\mathrm{N})$.

\begin{tabular}{|c|c|c|c|c|c|c|c|c|c|c|}
\hline \multirow{2}{*}{ Biofertilizante } & \multirow{2}{*}{$\mathrm{N}\left(\mathrm{kg} \mathrm{ha}^{-1}\right)$} & \multicolumn{3}{|c|}{$\overline{T C C}\left(\mathrm{~g} \mathrm{~m}^{-2} d^{-1}\right)$} & \multicolumn{3}{|c|}{$\overline{T A N}\left(\mathrm{~g} \mathrm{~m}^{-2} d^{-1}\right)$} & \multicolumn{3}{|c|}{$\mathrm{MS}\left(\mathrm{g} \mathrm{m}^{-2}\right)$} \\
\hline & & 31 & 75 & 105 & 31 & 75 & 105 & 31 & 75 & 105 \\
\hline & \multicolumn{10}{|c|}{ Días después de la siembra } \\
\hline & 0 & $6^{c}$ & $9^{a b}$ & $8^{c}$ & $25^{c}$ & $4.0^{a}$ & $2.5^{c}$ & $181^{c}$ & $618^{c}$ & $885^{c}$ \\
\hline \multirow[t]{3}{*}{$\operatorname{Sin}$} & 80 & $7^{c}$ & $9^{a b}$ & $7^{c}$ & $26^{c}$ & $3.5^{a}$ & $2.2^{c}$ & $206^{c}$ & $613^{c}$ & $840^{c}$ \\
\hline & 160 & $10^{b}$ & $10^{a b}$ & $8^{c}$ & $33^{b}$ & $3.2^{a}$ & $2.0^{c}$ & $278^{b}$ & $729^{b}$ & $985^{b}$ \\
\hline & 0 & $10^{b}$ & $8^{b}$ & $11^{b}$ & $35^{b}$ & $3.7^{a}$ & $4.5^{b}$ & $270^{b}$ & $643^{c}$ & $983^{b}$ \\
\hline \multirow[t]{2}{*}{ Con } & 80 & $12^{a}$ & $9^{a b}$ & $22^{a}$ & $38^{a b}$ & $3.5^{a}$ & $6.5^{a}$ & $317^{a}$ & $740^{b}$ & $1412^{a}$ \\
\hline & 160 & $12^{a}$ & $10^{a}$ & $20^{a}$ & $39^{a}$ & $3.7^{a}$ & $5.2^{b}$ & $339^{a}$ & $819^{a}$ & $1440^{a}$ \\
\hline \multicolumn{2}{|c|}{ Tukey $\alpha=0.05(\mathrm{DMS})$} & 1.4 & 1.3 & 2.3 & 3 & 0.9 & 1.1 & 51 & 69 & 445 \\
\hline \multicolumn{2}{|c|}{ Media general } & 10 & 9 & 13 & 33 & 3.6 & 3.8 & 265 & 693 & 1091 \\
\hline \multicolumn{2}{|c|}{ CV. $(\%)$} & 6 & 8 & 7 & 7 & 14 & 10 & 6 & 4 & 3 \\
\hline
\end{tabular}

y a la aplicación de 80 y $160 \mathrm{~kg} \mathrm{ha}^{-1} \mathrm{~N}$. Mientras que la EAN más baja la tuvo la aplicación de cero unidades de nitrógeno, no importanto si se aplicó o no aplicó biofertilizante.

\section{Análisis económico}

El análisis económico para el RG (Tabla 6), determinó que con la aplicación de Biofertilizante y $160 \mathrm{~kg} \mathrm{ha}^{-1}$ de nitrógeno, se tuvo el mayor ingreso neto (IN), al igual que el costo total (CT) y la 
Tabla 4. Índice de cosecha (IC), rendimiento de grano (RG) y sus componentes del maíz VS-535 en función del biofertilizante y nitrógeno $(\mathrm{N})$.

\begin{tabular}{|c|c|c|c|c|c|c|c|}
\hline Biofertilizante & $\mathrm{N}\left(\mathrm{kg} \mathrm{ha}{ }^{-1}\right)$ & IC (\%) & $\mathrm{RG}\left(\mathrm{g} \mathrm{m}^{-2}\right)$ & P100G (g) & $\mathrm{NH}$ & $\mathrm{NGH}$ & $N G\left(m^{2}\right)$ \\
\hline & 0 & $43^{b}$ & $386^{c}$ & $33^{a}$ & $18^{b}$ & $10^{a}$ & $1182^{b}$ \\
\hline \multirow[t]{3}{*}{$\operatorname{Sin}$} & 80 & $53^{a}$ & $443^{c}$ & $35^{a}$ & $20^{a b}$ & $10^{a}$ & $1342^{b}$ \\
\hline & 160 & $54^{a}$ & $533^{b}$ & $35^{a}$ & $20^{a b}$ & $11^{a}$ & $1397^{b}$ \\
\hline & 0 & $42^{b}$ & $417^{c}$ & $35^{a}$ & $19^{b}$ & $11^{a}$ & $1425^{b}$ \\
\hline \multirow[t]{2}{*}{ Con } & 80 & $40^{b}$ & $567^{b}$ & $38^{a}$ & $20^{a b}$ & $11^{a}$ & $1498^{a b}$ \\
\hline & 160 & $46^{b}$ & $657^{a}$ & $37^{a}$ & $22^{a}$ & $12^{a}$ & $1820^{a}$ \\
\hline \multicolumn{2}{|c|}{ Tukey $\alpha=0.05$} & 7 & 80 & 6 & 2 & 2 & 385 \\
\hline \multirow{2}{*}{\multicolumn{2}{|c|}{ Media general }} & 46 & 500 & 35 & 20 & 11 & 1444 \\
\hline & & 4 & 4 & 4 & 5 & 6 & 10 \\
\hline
\end{tabular}

En columnas para cada factor principal letras similares indican que los valores son estadísticamente iguales (Tukey, $\alpha=0.05$ ).

Tabla 5. Eficiencia en el uso del agua de la materia seca (EUAMS), rendimiento(EUARG) y eficiencia agronómica de nitrógeno (EAN) en el maíz VS-535 en función del biofertilizante y nitrógeno.

\begin{tabular}{|c|c|c|c|c|}
\hline Biofertilizante & $\begin{array}{l}\text { Nitrógeno } \\
\text { kg N ha-1 }\end{array}$ & $\begin{array}{c}\text { EUAMS } \\
\mathrm{g} \mathrm{m}^{-2} \mathrm{~mm}^{-1}\end{array}$ & $\begin{array}{c}\text { EUARG } \\
\mathrm{g} \mathrm{m}^{-2} \mathrm{~mm}^{-1}\end{array}$ & $\begin{array}{r}\text { EAN } \\
\mathrm{g} \mathrm{g}^{-1}\end{array}$ \\
\hline \multirow{4}{*}{$\operatorname{Sin}$} & 0 & $2.1^{c}$ & $0.9^{c}$ & $0^{c}$ \\
\hline & 80 & $2.1^{c}$ & $1.0^{c}$ & $7^{b}$ \\
\hline & 160 & $2.4^{b}$ & $1.3^{b}$ & $9^{b}$ \\
\hline & 0 & $2.4^{b}$ & $1.0^{c}$ & $0^{c}$ \\
\hline \multirow[t]{2}{*}{ Con } & 80 & $3.4^{a}$ & $1.3^{b}$ & $18^{a}$ \\
\hline & 160 & $3.5^{a}$ & $1.6^{a}$ & $15^{a}$ \\
\hline \multicolumn{2}{|c|}{ Tukey $\alpha=0.05$} & 0.1 & 0.07 & 2.7 \\
\hline \multicolumn{2}{|c|}{ Media general } & 2.6 & 1.2 & 8.3 \\
\hline \multicolumn{2}{|c|}{ CV. $(\%)$} & 3.1 & 3.9 & 23 \\
\hline
\end{tabular}

En columnas letras similares indican que los valores son estadísticamente iguales (Tukey, $\alpha=0.05$ ).

Tabla 6. Rendimiento de grano (RG), ingresos totales (IT), costos fijos (CF), costos variables (CV) y costos totales (CT), ingresos netos (IN) y ganancia por peso invertido (GPI) en la variedad VS-535 en función del biofertilizante y nitrógeno $(\mathrm{N})$.

\begin{tabular}{ccccccccc}
\hline Biofertilizante & $\begin{array}{c}\mathrm{N} \\
\left(\mathrm{kg} \mathrm{ha}^{-1}\right)\end{array}$ & $\begin{array}{c}\mathrm{RG} \\
\left(\mathrm{kg} \mathrm{ha}^{-1}\right)\end{array}$ & $\mathrm{IT}(\$)$ & $\mathrm{CF}(\$)$ & $\mathrm{CV}(\$)$ & $\mathrm{CT}(\$)$ & $\mathrm{IN}(\$)$ & $\mathrm{GPI}(\$)$ \\
\hline \multirow{3}{*}{ Sin } & 0 & $3860^{c}$ & 15440.00 & 5033.60 & 1150.00 & 6183.60 & 9256.40 & 1.50 \\
& 80 & $4430^{c}$ & 17720.00 & 5033.60 & 2673.00 & 7706.60 & 10013.40 & 1.30 \\
& 160 & $5330^{b}$ & 21320.00 & 5033.60 & 4096.00 & 9129.60 & 12190.40 & 1.34 \\
\multirow{4}{*}{ Con } & 0 & $4170^{c}$ & 16680.00 & 5033.60 & 1742.00 & 6775.60 & 9904.40 & 1.46 \\
& 80 & $5670^{b}$ & 22680.00 & 5033.60 & 3265.00 & 8298.60 & 14381.40 & 1.70 \\
& 160 & $6570^{a}$ & 26280.00 & 5033.60 & 4688.00 & 9721.60 & 16558.40 & 1.73 \\
\hline
\end{tabular}

IT $=$ RG * precio por kg de maíz (\$4.00). CF = incluye costo de preparación del terreno, siembra, riego, manejo de maleza y plagas. $\mathrm{CV}=$ incluye el costo de la fertilización y cosecha de maíz. $\mathrm{CT}=\mathrm{CF}+\mathrm{CV}$. IN $=\mathrm{IT}-\mathrm{CT}$. $\mathrm{GPI}=\mathrm{IN} / \mathrm{CT}$.

ganancia por peso invertido (GPI). Para cada peso invertido, se recuperaron $\$ 1.73$.

\section{DISCUSIÓN}

Las condiciones climáticas en las que se desarrolló el cultivo fue el adecuado, debido a que la temperatura fluctuó entre 34 y $24^{\circ} \mathrm{C}$. Dichos va- lores se encuentran dentro del umbral térmico promedio de 30 a $34^{\circ} \mathrm{C}$ apropiado para el cultivo de maíz en el área de estudio (Aguilar et al. 2015b). La precipitación fue de $558 \mathrm{~mm}$, valor similar al señalado por Rivetti (2006) quien indica que la necesidad de agua por ciclo de cultivo del maíz es de $575 \mathrm{~mm}$. La variedad estudiada tuvo un requerimiento de calor de 3096 UC desde la 


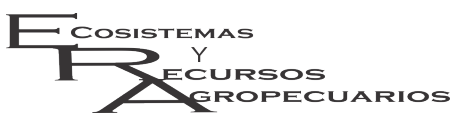

emergencia hasta la madurez fisiológica. Lo que difiere con Díaz et al. (2013) quienes reportan un requerimiento térmico de 1158 UC para el cultivo de maíz en clima templado, valor inferior al encontrado en el presente estudio, debido a que en climas tropicales las temperaturas son superiores.

A los $30 \mathrm{~d}$ de haber iniciado el estudio se observó que la aplicación del biofertilizante promovió la aparición de las hojas, lo que genero un mayor IAF, MS, TCC, TAN, lo que repercutió en los componentes del rendimiento (P100G, NH, NGH y NG), lo que sugiere que el suministro de biofertilizante indujo un estímulo en el crecimiento y rendimiento del maíz (Roveda y Polo 2007). Por otro lado, el biofertilizante fue favorecido con la incorporación de nitrógeno, desde los 30 dds la fertilización con $160 \mathrm{~kg} \mathrm{ha}^{-1} \mathrm{~N}$ tuvo un incrementó en la aparición de hoja e IAF, lo que generó los mayores índices de crecimiento (TAN, TCC), así como el mayor peso de planta, como lo han reportado en maíz Naresh y Singh (2001), Loredo et al. (2004) y García et al. (2007). Cabe señalar, que con la dosis más alta de nitrógeno y biofertilizante, se logró incrementar la eficiencia en el uso del agua para la materia seca y rendimiento, lo que sugiera que la planta optimiza el agua uso del agua (Caviglia y Sadras 2001). La variedad en estudio presentó la mejor eficiencia agronómica del nitrógeno con la aplicación de biofertilizante, sin embargo, al reducir la fertilización nitrogenada $\left(80 \mathrm{~kg} \mathrm{ha}^{-1} \mathrm{~N}\right)$, la EAN fue más alto. Lo que, indica el potencial del genotipo en la asimilación del nitrógeno (Aguilar et al. 2015b), debido al biofertilizante (Díaz et al. 2008).

El análisis económico para el rendimiento presentado se observa que con la fertilización nitro-
Aguilar-Carpio et al. Análisis de crecimiento en maíz Ecosist. Recur. Agropec. 4(12):475-483,2017 genada en asociación con el biofertilizante se generó el mayor ingreso neto, costo total y la ganancia por peso invertido. Por cada peso invertido, se recuperaron \$1.73. Para los agricultores de capital limitado, que siembren la variedad VS-535, se recomienda el uso de biofertilizante, debido a que genera el mayor ingreso neto sin aplicación de nitrógeno $(\$ 9,904.40)$. Lo que concuerda con lo reportado por Armenta et al. (2010), quienes reportan que el uso del biofertilizante asociados con nitrógeno, incrementa la rentabilidad del maíz.

\section{CONCLUSIONES}

En las condiciones ambientales en que se desarrolló el cultivo, con $160 \mathrm{~kg} \mathrm{ha}^{-1}$ de nitrógeno se tuvo la mayor respuesta en el IAF y DAFT a los 75 dds. Para la MS, $\overline{T C C}$ y $\overline{T A N}$, los valores más altos se tuvieron con la aplicación de biofertilizante, y la aplicación de 80 y $160 \mathrm{~kg} \mathrm{ha}^{-1}$ de nitrógeno, durante el ciclo del cultivo. Para RG la mayor respuesta al $\mathrm{N}$ se encontró con la aplicación de 160 $\mathrm{kg} \mathrm{ha}^{-1}$, más el biofertilizante. La aplicación de biofertilizante con 80 y $160 \mathrm{~kg} \mathrm{ha}^{-1}$ de nitrógeno, incrementó la eficiencia en el uso del agua, eficiencia agronómica de nitrógeno, producción de materia seca y rendimiento de grano. La mejor rentabilidad se logra con biofertilizante y $160 \mathrm{~kg} \mathrm{ha}^{-1}$ de nitrógeno.

\section{AGRADECIMIENTOS}

Al pueblo de México que mediante CONACYT otorgó el apoyo económico para llevar a cabo este trabajo de investigación.

\section{LITERATURA CITADA}

Aguilar CC, Escalante EJAS, Aguilar MI (2015a) Análisis de crecimiento y rendimiento de maíz en clima cálido en función del genotipo, biofertilizante y nitrógeno. Terra Latinoamericana 33: 51-62.

Aguilar CC, Escalante EJAS, Aguilar MI, Mejía CJA, Conde MVF, Trinidad SA (2015b) Rendimiento y rentabilidad de maíz en función del genotipo, biofertilizante y nitrógeno, en clima cálido. Tropical and Subtropical Agroecosystems 18: 151-163.

Andrade F, Echarte L, Rizzalli R, Della Maggiora A, Casanovas M (2002) Kernel number prediction in maize 
under nitrogen or water stress. Crop Science 42: 1173-1179.

Armenta BAD, García CG, Camacho JRB, Apodaca MAS, Montoya LG, Nava EP (2010) Biofertilizante en el desarrollo agrícola de México. Ra Ximhai 6: 51-56.

Caviglia OP, Sadras VO (2001) Effect of nitrogen supply on crop conductance, water- and radiation-use efficiency of wheat. Field Crops Research 69: 259-266.

D'Andrea KE, Otegui ME, Cirilo AG (2008) Kernel number determination differs among maize hybrids in response to nitrogen. Field Crops Research 105: 228-239.

Díaz FA, Salinas JRG, Garza IC, Mayek NP (2008) Impacto de labranza e inoculación micorrízica arbuscular sobre la pudrición carbonosa y rendimiento de maíz en condiciones semiáridas. Revista Fitotecnia Mexicana 31: 257-263.

Díaz LE, Loeza-Corte JM, Campos-Pastelín JM, Morales-Rosales EJ, Domínguez-López A, Franco-Mora O (2013) Eficiencia en el uso de la radiación, tasa de asimilación neta e integral térmica en función del fósforo en maíz (Zea mays L.). Agrociencia 47:135-146.

Domínguez MCA, Brambila PJJ, Carballo CA, Quero CAR (2014) Red de valor para maíz con alta calidad de proteína. Revista Mexicana de Ciencias Agrícolas 5: 391-403.

Doorenbos J, Pruitt WO (1986) Las necesidades de agua por los cultivos. Estudio FAO. Riego y Drenaje. Manual 24. FAO. Roma. 144p.

Escalante EJAS (1995) Aprovechamiento del recurso agua en cultivos de secano. Agroproductividad 12: 28-32.

Espinosa A, Sierra M, Gómez N (2003) Producción y tecnología de semillas mejoradas de maíz por el INIFAP en el escenario sin la PRONASE. Agronomia Mesoamericana 14:117-121.

Fageria NK, Baligar VC (2005) Enhancing nitrogen use efficiency in crop plants. Advances in Agronomy 88: 97-185.

García E (2005) Modificación al sistema de clasificación climática de Köppen. $4^{a}$ Edición. Instituto de Geografía. Universidad Autónoma de México. 217p.

García OJG, Moreno VRM, Rodríguez ICL, Mendoza AH, Mayer NP (2007) Efecto de cepas de Azospirillum brasilense en el crecimiento y rendimiento de grano del maíz. Revista Fitotecnia Mexicana 30: 305-310.

Íñiguez-Covarrubias M, Ojeda-Bustamante W, Díaz-Delgado C, Sifuentes-Ibarra E (2014) Análisis de cuatro variables del período de lluvias asociadas al cultivo maíz de temporal. Revista Mexicana de Ciencias Agrícolas 5: 101-114.

López PP, Prieto PFG, Gaytán MM, Román ADG (2007) Caracterización fisicoquímica de diferentes variedades de cebada cultivadas en la Región Centro de México. Revista Chilena Nutrición 34: 1-12.

Loredo OC, López RL, Espinosa VD (2004) Bacterias promotoras del crecimiento vegetal asociadas con gramíneas: una revisión. Terra Latinoamericana 22: 225-239.

Mamani-Pati F, Clay DE, Carlson CG, Clay AS, Reicks G, Kim K (2010) Nitrogen rate, landscape position and harvesting of corn stover impacts on energy gains and sustainability of corn production systems in South Dakota. Agronomy Journal 102: 1535-1541.

Naresh KS, Singh CP (2001) Growth analysis of maize during long and short duration crop seasons: influence of nitrogen source and dose. Indian Journal of Agricultural Research 35: 13-18. 
Pecina QV, Díaz FA, Williams AH, Rosales RE, Garza CI (2005) Influencia de fechas de siembra y biofertilizantes en sorgo. Revista Fitotecnia Mexicana 28: 389-392.

Ritchie SW, Hanway JJ (1982) How a corn plant develops. lowa State University of Science and Technology. Cooperative Extension Service. No. 48. Ames lowa, USA. 21p.

Rivetti A (2006) Producción de maíz bajo diferentes regímenes de riego complementario en Rio Cuarto, Córdoba, Argentina. I. Rendimiento en grano de maíz y sus componentes. Revista de la Facultad de Ciencias Agrarias 38: 25-36.

Roveda G, Polo C (2007) Mecanismos de adaptación de maíz asociado a Glomus spp. En suelos con bajo fósforo disponible. Agronomía colombiana 25: 349-356.

Sistema de Información Agropecuaria SIAP (2016) Sistema de Información Agropecuaria de Consulta. SAGARPA. México. Disponible en: http://www.siap.sagarpa.gob/mx/ar_comanuar.html. Fecha de consulta 29 de mayo de 2017.

Smith SE, Read DJ (2008) Mycorrhizal Simbiosis. 3th Ed. Academic Press. NY, USA. 605p.

Snyder RL (1985) Hand calculating degree-days. Agricultural and Forest Meteorology 35: 353-358.

Uribe VG, Dzib ER (2006) Micorriza arbuscular (Glomus intraradices), Azospirillum brasilense y Brassinoesteroide, en la producción de maíz en suelo luvisol. Agricultura Técnica en México 32: 68-72.

Villafaña ATL, Morales RJE, Estrada CG, Martínez RGC (2014) Determinantes ecofisiológicos del rendimiento en tres cultivares de trigo en función y fraccionamiento del nitrógeno. Revista Mexicana de Ciencias Agrícolas 5: 405-419.

Volke HV (1982) Optimización de insumos de la producción en la agricultura. Colegio de Postgraduados. Chapingo. México. 61p.

Yu-kui R, Shi-ling J, Fu-Sou Z, Jian-Bo S (2009) Efectos de la aplicación de fertilizantes nitrogenado en la composición de los elementos en los granos de maíz. Agrociencia 43: 21-27. 
